\title{
Comparative studies of methods of obtaining AGW's propagation properties
}

\author{
H. Y. Lue ${ }^{1}$ and F. S. Kuo ${ }^{2}$ \\ ${ }^{1}$ Department of Physics, Fu Jen University, Hsin Chuang, Taiwan \\ ${ }^{2}$ Department of Electro-Optical Engineering, Vanung University, Chung-Li, Taiwan \\ Correspondence to: F. S. Kuo (fushongkuo@gmail.com) \\ Received: 25 January 2011 - Revised: 5 January 2012 - Accepted: 10 February 2012 - Published: 19 March 2012
}

\begin{abstract}
Three among the existing methods of obtaining the properties (intrinsic period, wavelength, propagation direction) of atmospheric gravity waves (AGWs) were compared and studied by numerical method to simulate radar data. Three-dimensional fluctuation velocity satisfying dispersion equation and polarization relation of atmospheric gravity wave were generated, then the numerical data were analysed by these methods to obtain the properties of waves. We found that, hodograph analysis was accurate for a monochromatic wave in obtaining its wave period and propagation direction, but the analysis became erratic for the case of multiple waves' superposition. The error was especially large when data consisted of both upward propagating waves and downward propagating waves. The hodograph method became meaningful again if all the component waves propagated in the same direction and the resulting period was dominantly decided by the lowest frequency wave. Stokes parameters method would obtain statistically meaningful values of wave period and azimuth if the spreading of the azimuths among the component waves did not exceed $90^{\circ}$ and the resulting period and azimuth were dominated by the lowest frequency wave component as well, irrespective of the vertical sense of propagation. Another method called phase and group velocity tracing technique was reconfirmed to be meaningful in measuring the characteristic wave period and vertical group and phase velocities of a wave packet: the characteristic wave period and vertical wavelength was dominated by the wave with the highest frequency among the component waves in the wave packet. Based on these numerical results, a composite procedure of data analysis for wave propagation was proposed and an example of real data analysis was presented.
\end{abstract}

Keywords. Meteorology and atmospheric dynamics (Middle atmosphere dynamics)

\section{Introduction}

There are many methods to obtain propagation properties of atmospheric gravity waves (AGWs), three of them hold our current interest: hodograph method, Stokes parameters method and the technique of phase and group velocity tracing. Hodograph analysis on a single monochromatic atmospheric gravity wave is accurate in obtaining its intrinsic frequency and propagation direction. According to polarization relation of gravity wave (in Northern Hemisphere), if the wave has a downward (upward) phase velocity, its hodograph-ellipse will have a clockwise (counter-clockwise) rotation, the ratio of the major to minor axis equals the frequency ratio $\omega / f$ of intrinsic frequency $\omega$ to inertial frequency $f$, the major axis lies along the horizontal propagation direction of the wave (Hirota and Niki, 1985; Nakamura et al., 1993; Tsuda et al., 1990), and the $180^{\circ}$-ambiguity of the horizontal propagation direction (along the major axis of the hodograph) can be solved by a relation between the horizontal and vertical perturbation velocities (Tsuda et al., 1990). Practically, monochromatic wave is extremely hard to get, so Stokes parameters method was proposed (Vincent and Fritts, 1987) to calculate the propagation parameters (intrinsic wave period and horizontal oscillation direction) of gravity waves statistically. Comparison of hodograph and Stokes parameters analysis was provided by Eckermann (1996). In contrast to the case of monochromatic wave, there is no simple analytic formula to describe the hodograph and Stokes parameters for the case of multiple waves' superposition, so numerical simulation is required to examine these methods. The third method called phase and group velocity tracing (see Sect. III of Kuo et al., 2003) is accurate in measuring the phase and group velocity of a wave packet along a radar beam. When the dual beam method is applied, the locations (time and height), the vertical phase and group velocities as 
well as the characteristic wave periods of wave packets can be obtained. Then the most probable characteristic intrinsic frequency, horizontal wavelength and azimuth of each wave packet can be found by fitting the dispersion equation and its related formula for vertical group velocity (Kuo et al., 2009). In this paper, we shall compare the merit and demerit of each method and try to find a composite procedure for obtaining the most probable propagation parameters of AGWs. One example of wave packet analysis of real radar data will be presented to demonstrate the procedure.

\section{Formulation of gravity wave-induced fluctuation ve- locities and Stokes parameters}

The gravity wave induced 3-D fluctuation velocities must satisfy its Doppler relation (1a) and dispersion Eq. (1b) (Fritts and Alexander, 2003),

$\omega=\sigma-\boldsymbol{k} \cdot \boldsymbol{u}_{0}$,

$m^{2}=\frac{\left(k^{2}+\ell^{2}\right)\left(N^{2}-\omega^{2}\right)}{\left(\omega^{2}-f^{2}\right)}-\frac{1}{4 H^{2}}$,

and polarization relation (2a, 2b) (Gossard and Hooke, 1975; see also Kuo et al., 2009),

$$
\begin{aligned}
W & =\frac{i \omega}{N^{2}-\omega^{2}}\left(\frac{\omega^{2}-f^{2}}{\omega k+i f \cdot \ell}\right)\left(\frac{\partial}{\partial z}+\Gamma\right) U \\
& =\frac{\omega\left(\omega^{2}-f^{2}\right)}{N^{2}-\omega^{2}} \sqrt{\frac{m^{2}+\Gamma^{2}}{\omega^{2} k^{2}+f^{2} \ell^{2}}} \times e^{i\left(\theta_{1}+\theta_{2}\right)} \times U, \\
W & =\frac{i \omega}{N^{2}-\omega^{2}}\left(\frac{\omega^{2}-f^{2}}{\omega \ell-i f \cdot k}\right)\left(\frac{\partial}{\partial z}+\Gamma\right) V \\
& =\frac{\omega\left(\omega^{2}-f^{2}\right)}{N^{2}-\omega^{2}} \sqrt{\frac{m^{2}+\Gamma^{2}}{\omega^{2} \ell^{2}+f^{2} k^{2}}} \times e^{i\left(\theta_{3}+\theta_{2}\right)} \times V,
\end{aligned}
$$

or inversely,

$$
\begin{aligned}
& U=\frac{N^{2}-\omega^{2}}{\omega\left(\omega^{2}-f^{2}\right)} \sqrt{\frac{\omega^{2} k^{2}+f^{2} \ell^{2}}{m^{2}+\Gamma^{2}}} \times e^{-i\left(\theta_{1}+\theta_{2}\right)} \times W, \\
& V=\frac{N^{2}-\omega^{2}}{\omega\left(\omega^{2}-f^{2}\right)} \sqrt{\frac{\omega^{2} \ell^{2}+f^{2} k^{2}}{m^{2}+\Gamma^{2}}} \times e^{-i\left(\theta_{3}+\theta_{2}\right)} \times W .
\end{aligned}
$$

Here $\boldsymbol{u}_{0}$ is the background wind velocity, $\boldsymbol{k}$ is the horizontal wave vector; $U, V$ and $W$ are the amplitudes of zonal, meridional and vertical velocities, respectively; $\sigma, \omega, k, \ell$ and $m$, respectively, are the observed frequency, intrinsic frequency, zonal-, meridional-, and vertical- wave number component. $\theta_{1}=\tan ^{-1}\left(\frac{\omega \cdot k}{f \cdot \ell}\right), \theta_{2}=\tan ^{-1}\left(\frac{m}{\Gamma}\right), \theta_{3}=\tan ^{-1}\left(\frac{\omega \cdot \ell}{-f \cdot k}\right)$. $\Gamma \cong 3.2 \times 10^{-5} \mathrm{rad} / \mathrm{m}$ (Eckart's coefficient), $N=2.09 \times$ $10^{-2} \mathrm{rad} / \mathrm{s}$ (Brunt-Vaisala frequency, corresponding to $5 \mathrm{~min}$ period), $f \cong 8.31 \times 10^{-5} \mathrm{rad} / \mathrm{s}$ (inertial frequency, corresponding to $21 \mathrm{~h}$ period, this value would exist for the latitude $30^{\circ} \mathrm{N}$ ) are assumed throughout this study. Then the vertical, zonal, and meridional components of fluctuation velocities $w, u$ and $v$ of a wave mode with intrinsic frequency $\omega$, observed frequency $\sigma$, wave numbers $m, k$ and $\ell$ were given by,

$w=W \cos (k x+\ell y+m z-\sigma \cdot t)$,

$$
\begin{aligned}
u= & U \cos (k x+\ell y+m z-\sigma \cdot t) \\
= & \frac{N^{2}-\omega^{2}}{\omega\left(\omega^{2}-f^{2}\right)} \sqrt{\frac{\omega^{2} k^{2}+f^{2} \ell^{2}}{m^{2}+\Gamma^{2}}} \\
& \cdot W \cos \left(k x+\ell y+m z-\sigma \cdot t-\theta_{1}-\theta_{2}\right), \\
v= & V \cos (k x+\ell y+m z-\sigma \cdot t) \\
= & \frac{N^{2}-\omega^{2}}{\omega\left(\omega^{2}-f^{2}\right)} \sqrt{\frac{\omega^{2} \ell^{2}+f^{2} k^{2}}{m^{2}+\Gamma^{2}}} \\
& \cdot W \cos \left(k x+\ell y+m z-\sigma \cdot t-\theta_{3}-\theta_{2}\right),
\end{aligned}
$$

and,

$$
\begin{aligned}
\tilde{u}= & U \cos \left(k x+\ell y+m z-\sigma \cdot t-90^{\circ}\right) \\
= & \frac{N^{2}-\omega^{2}}{\omega\left(\omega^{2}-f^{2}\right)} \sqrt{\frac{\omega^{2} k^{2}+f^{2} \ell^{2}}{m^{2}+\Gamma^{2}}} \\
& \cdot W \sin \left(k x+\ell y+m z-\sigma \cdot t-\theta_{1}-\theta_{2}\right),
\end{aligned}
$$

where $\tilde{u}$ is a $90^{\circ}$ phase shift of zonal fluctuation velocity and was prepared for Stokes parameters analysis. The Stokes parameters were given by,

$$
\begin{aligned}
& I=\left\langle\overline{u^{2}}+\overline{v^{2}}\right\rangle, \\
& D=\left\langle\overline{u^{2}}-\overline{v^{2}}\right\rangle, \\
& P=2\langle\overline{u v}\rangle, \\
& Q=2\langle\overline{\tilde{u} v}\rangle,
\end{aligned}
$$

where overbar denoted average over time $t$ and angle bracket $(<>)$ represented average over height $z$. In optical terms, $I$ is the throughout parameter, $D$ is the throughout anisotropic parameter, $P$ is the linear polarization parameter and $Q$ is the circular polarization parameter.

\section{Analysis of simulation data}

Data generated from various models were analysed by the methods mentioned in Sect. 1. For simplicity of discussion, we would focus on the cases with negligible background wind in which $u_{0} \cong 0$ and $\omega \cong \sigma$. 


\subsection{Analysis of a single monochromatic wave}

We generated vertical profiles of three wind components as well as the horizontal speed induced by a monochromatic wave (with downward phase velocity) characterised by

$\tau=8 h, \quad \lambda_{\mathrm{h}}=900 \mathrm{~km}, \quad \varphi_{\mathrm{az}}=-45^{\circ}, \quad \lambda_{\mathrm{z}}=-8.70 \mathrm{~km}$.

(Parameters A)

Here $\tau=2 \pi / \omega$ is the wave period, $\lambda_{\mathrm{h}}$ is the horizontal wavelength, $\phi_{\mathrm{az}}$ is the azimuth of the wave vector, and $\lambda_{\mathrm{z}}$ is the vertical wavelength. A negative vertical wavelength $\lambda_{z}$ represents a downward phase velocity (upward group velocity). Our analysis (not shown) confirmed that hodograph method was perfect for the case of a single monochromatic wave at each time step. This monochromatic wave data cannot be analysed by phase and group velocity tracing technique which is a method to analyse wave packet only.

In the following, we have a look at the results from the Stokes parameters method. The phase difference $\delta\left(=\theta_{3}-\right.$ $\left.\theta_{1}\right)$, major axis orientation $\phi\left(=90^{\circ}-\phi_{\mathrm{az}}\right)$, the degree of polarization $d$ and ellipse axis ratio AR were given by (Vincent and Fritts, 1987; Eckermann and Vincent, 1989),

$\delta=\arctan (Q / P)$,

$2 \phi=\arctan (P / D)$,

$d=\left(D^{2}+P^{2}+Q^{2}\right)^{1 / 2} / I$

$\mathrm{AR}=\cot \xi$ where $2 \xi=\arcsin \left(\frac{Q}{d \cdot I}\right)$.

The intrinsic wave period was found using

$\tau=\frac{2 \pi}{f \cdot \mathrm{AR}}$.

It is clear that Eq. (6b) cannot distinguish between the major axis orientation $\phi$ and $\phi \pm 180^{\circ}$, implying that Stokes parameters analysis cannot distinguish between eastward (northward) wave and westward (southward) wave. Also, Eqs. (6a)-(6e) cannot distinguish a phase-upward from a phase-downward propagating wave. The Stokes parameters analysis of oscillation data of the gravity wave characterised by Parameters A yielded that $\tau=8 \mathrm{~h}, \varphi_{\mathrm{az}}=-45^{\circ}$, and $d=1$, at each height. When the vertical sense of phase propagation of the wave in Parameters A was changed from downward to upward, or its azimuth was changed from $-45^{\circ}$ to $135^{\circ}$, we obtained exactly same result ( $\tau=8 \mathrm{~h}, \varphi_{\mathrm{az}}=-45^{\circ}$ and $d=1$, at each height) as expected. In general practice, the $180^{\circ}$ ambiguity in horizontal propagation direction can be solved by the correlation with simultaneous measurements of temperature oscillations (Kitamura and Hirota, 1989; Hamilton, 1991) due to the polarization between temperature, zonal and meridional wind (see e.g., Fritts and Alexander, 2003).
Table 1a. Properties of the AGWs to be superposed to generate the perturbation velocities. Note: The negative sign of $\lambda_{z}$ means downward phase propagation.

\begin{tabular}{cccccc}
\hline$j$ & $\tau(\mathrm{h})$ & $\lambda_{\mathrm{h}}(\mathrm{km})$ & $\varphi_{\mathrm{az}}\left(^{\circ}\right)$ & $\lambda_{\mathrm{z}}(\mathrm{km})$ & $A_{j}$ \\
\hline 1 & 8 & 900 & 75 & -8.701 & 0.1 \\
2 & 8 & 900 & 75 & +8.701 & $0.1 \alpha$ \\
\hline
\end{tabular}

Table 1b. Same as Table 1a, but with different azimuth angles.

\begin{tabular}{cccccc}
\hline$j$ & $\tau(\mathrm{h})$ & $\lambda_{\mathrm{h}}(\mathrm{km})$ & $\varphi_{\mathrm{az}}\left({ }^{\circ}\right)$ & $\lambda_{\mathrm{z}}(\mathrm{km})$ & $A_{j}$ \\
\hline 1 & 8 & 900 & 75 & -8.701 & 0.1 \\
2 & 8 & 900 & 35 & +8.701 & $0.1 \alpha$
\end{tabular}

Table 1c. Same as Table 1b, but with identical vertical sense of propagation.

\begin{tabular}{cccccc}
\hline$j$ & $\tau(\mathrm{h})$ & $\lambda_{\mathrm{h}}(\mathrm{km})$ & $\varphi_{\mathrm{az}}\left({ }^{\circ}\right)$ & $\lambda_{\mathrm{z}}(\mathrm{km})$ & $A_{j}$ \\
\hline 1 & 8 & 900 & 75 & -8.701 & 0.1 \\
2 & 8 & 900 & 35 & -8.701 & $0.1 \alpha$ \\
\hline
\end{tabular}

\subsection{Superposition of two waves with opposite vertical propagation}

In the case of multiple waves' superposition, the perturbation velocity is the vector sum of perturbation velocities of all the waves. So zonal, meridional, vertical perturbation velocities $(u, v, w)$ and the $90^{\circ}$ phase shift of zonal fluctuation velocity $\tilde{u}$ of the superposed waves were given by

$u=\sum_{j} A_{j} u_{j}$

$v=\sum_{j} A_{j} v_{j}$

$w=\sum_{j} A_{j} w_{j}$

$\tilde{u}=\sum_{j} A_{j} \tilde{u}_{j}$

with $A_{j}$ being the wave amplitudes. Now let us consider a special case of superposition of 2 AGWs whose properties were listed in Table 1a, and the perturbation velocities were given in Eq. (8a)-(8d),

$$
\begin{aligned}
& u=0.1 u_{1}+0.1 \alpha u_{2}, \\
& v=0.1 v_{1}+0.1 \alpha v_{2}, \\
& w=0.1 w_{1}+0.1 \alpha w_{2},
\end{aligned}
$$



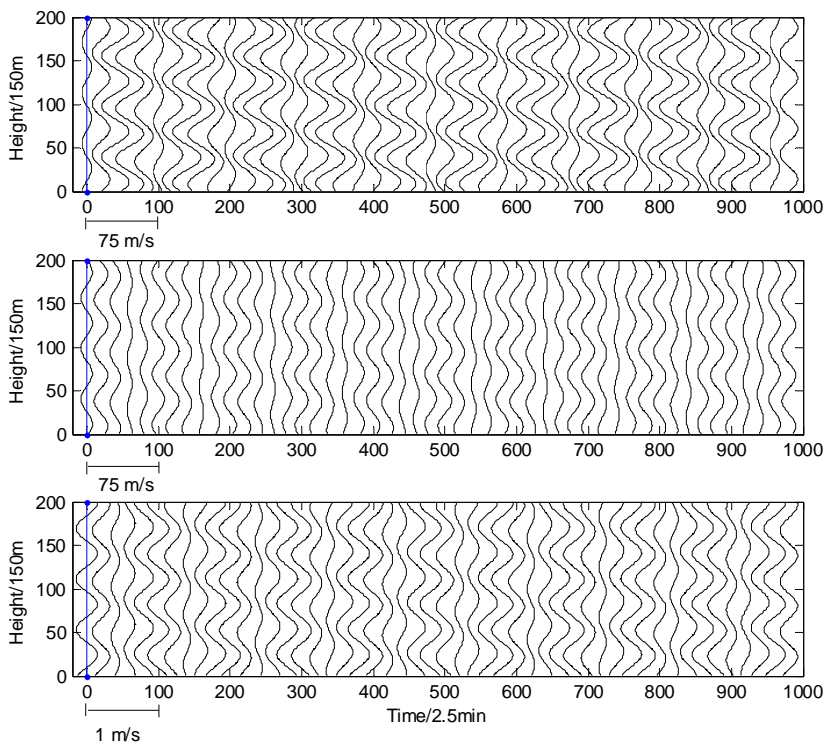

Fig. 1. Height variations of perturbation velocities at successive times. Top panel: zonal velocity profile; middle panel: meridional velocity profile; bottom panel: vertical velocity profile. The data were obtained from the superposition of two monochromatic waves characterised in Table 1a with $\alpha=0.5$ in Eqs. (8a), (8b), (8c). The time step is $2.5 \mathrm{~min}$ and the height resolution is $150 \mathrm{~m}$. The vertical lines indicated $0 \mathrm{~m} \mathrm{~s}^{-1}$ for the first profile and successive profiles are $50 \mathrm{~min}$ apart. The velocity scales are indicated below each panel.

$\tilde{u}=0.1 \tilde{u}_{1}+0.1 \alpha \tilde{u}_{2} \quad$.

Notice that these 2 component waves in Table 1a had the same wave period, wavelength and azimuth angle, but had opposite vertical phase propagation. The major component wave $(j=1)$ in Table 1a had downward phase velocity and the minor component wave $(j=2)$ had upward phase velocity. The free parameter $\alpha$ is the amplitude ratio of minor to major component wave. We would present and compare three cases of $\alpha=0.8, \alpha=0.5$ and $\alpha=0.25$. Again, this artificial data was not suitable for analysis by phase and group velocity tracing technique because there were too few waves to form credible wave packet.

\subsubsection{Wave analysis by hodograph method}

An example of the vertical profiles of zonal, meridional and vertical velocity obtained from Eqs. (8a), (8b), (8c) with amplitude ratio $\alpha=0.5$ shown on the top, middle and bottom panel, respectively, in Fig. 1, which clearly revealed downward phase propagation in all three panels. Their hodograph analyses were also made systematically at each time step. Almost all the hodographs could be perfectly fitted by ellipses, among them, two examples of hodographs analysed at the 85th and 35th time step were presented in Fig. 2a and $\mathrm{b}$, respectively, both hodographs had clockwise rota-

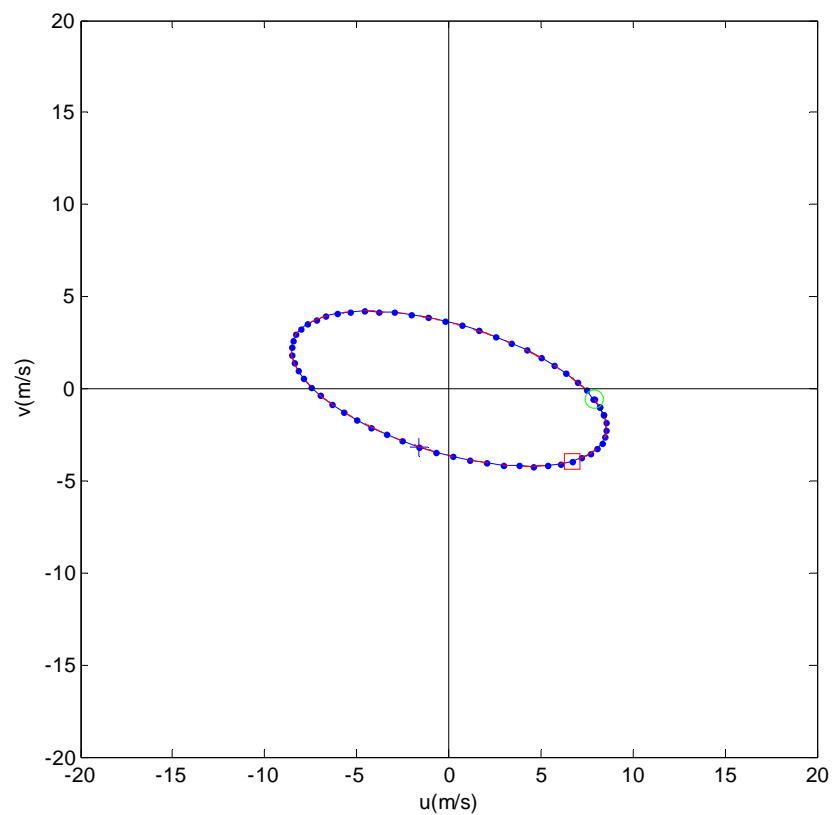

Fig. 2a. Hodograph analysis of vertical profiles of three wind components and the horizontal velocity amplitude induced by the superposition of two waves characterised by Table 1 a with $\alpha=0.5$ in Eqs. (8a), (8b), (8c) at the 85th time step. The rotation sense of this hodograph is clockwise (from a green circle to a red square to a blue cross). $\tau=8.25 \mathrm{~h}$ and $\phi_{\mathrm{az}}=106.6^{\circ}$ were obtained by this analysis.

tion. Hodograph analysis in Fig. 2a resulted in $\tau=8.25 \mathrm{~h}$ and $\phi_{\mathrm{az}}=106.6^{\circ}$, and the corresponding analysis of Fig. $2 \mathrm{~b}$ resulted in $\tau=3.17 \mathrm{~h}$ and $\phi_{\mathrm{az}}=65.4^{\circ}$. These two examples revealed that either the wave period $(3.17 \mathrm{~h}$ in Fig. $2 \mathrm{~b})$ or the azimuth $\left(106.6^{\circ}\right.$ in Fig. 2a) obtained by hodograph analysis was far too different from the true values (i.e., $\tau=8 \mathrm{~h}$, $\phi_{\mathrm{az}}=75^{\circ}$ ). A detailed hodograph analysis at each time step of Fig. 1 was summarized in Fig. 3, which showed the period vs. azimuth plots of all the results of hodograph analysis (by cross) along with the corresponding results of two other cases with $\alpha=0.8$ (represented by open circle) and $\alpha=0.25$ (represented by dot) and the original component waves (by upward triangle and downward triangle). To distinguish the direction of vertical propagation, the event with downward phase velocity was represented by a black symbol, while the event with upward phase velocity was represented by a red symbol. Figure 3 clearly demonstrated that the vertical propagation of all the resulting waves under study were the same as their major component wave (in terms of amplitude), i.e., downward phase propagation. Even if the amplitude ratio $\alpha$ was as large as 0.8 , the vertical sense of propagation of the resulting wave was still completely dominated by the major wave. Also, the error in the azimuth and wave period resulted from hodograph analysis decreased with decreasing $\alpha$, and the error was so large that even if this amplitude ratio was as small as 0.25 , the error was still too large to be acceptable. 


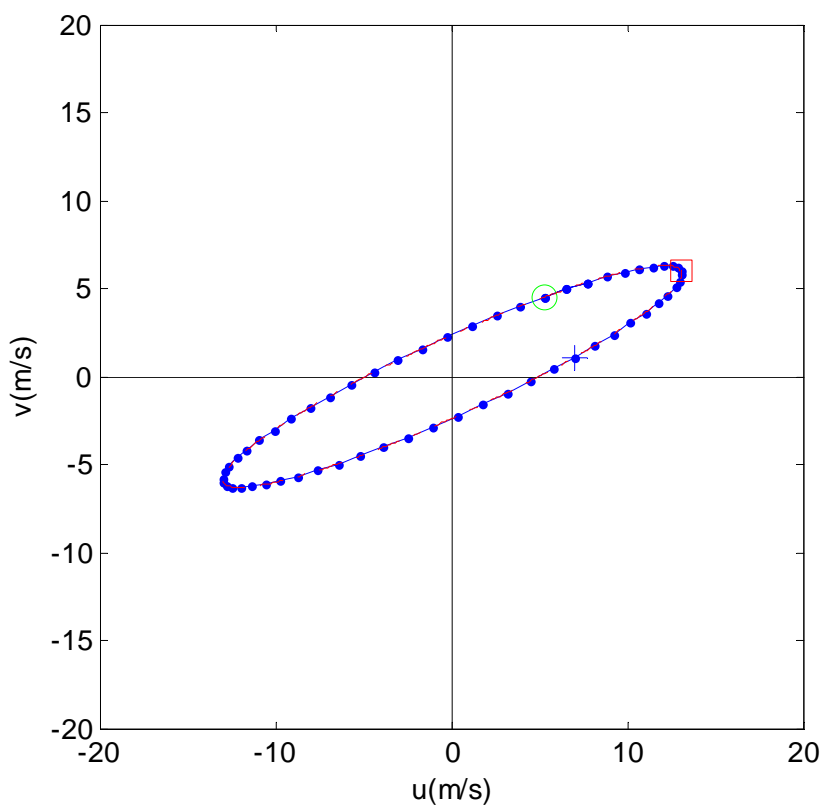

Fig. 2b. Same as Fig. 2a except at the 35th time step. The rotation sense of this hodograph is clockwise (from a green circle to a red square to a blue cross). $\tau=3.17 \mathrm{~h}$ and $\phi_{\mathrm{az}}=65.4^{\circ}$ were obtained by this analysis.

From the examples above, we concluded that superposition of two waves with the same period and wavelengths, but opposite vertical propagation would misleadingly yield hodographs perfectly fitted by ellipses with erroneous wave period and azimuth even though they propagated in same horizontal direction. Further studies showed that, additional error would result if the azimuths of these two component waves also differed. For example, a difference of 40 degrees in azimuths between the two waves as defined in Table 1b would contribute an additional $1 \mathrm{~h}$ of error in wave period and 10 degrees of error in azimuth.

\subsubsection{Wave analysis by Stokes parameters method}

The results of Stokes parameters analyses of three cases of zonal and meridional velocities, which were defined by Eqs. (8a), (8b), (8d) and Table 1a with amplitude ratio $\alpha=$ $0.8,0.5$, and 0.25 , were all the same at each height (without doing height averages in Eqs. 5a, 5b, 5c, 5d): $\tau=8 \mathrm{~h}, \varphi_{\mathrm{az}}=$ $75^{\circ}$ and $d=1$, which was exactly the same as a monochromatic wave in Table 1a irrespective of its vertical sense of propagation. This result was a natural consequence of Stokes parameters analysis because it could not identify the vertical sense of gravity wave propagation. When the azimuths of the two waves were separated by $40^{\circ}$ as shown in Table $1 \mathrm{~b}$, the results of Stokes parameters analyses were as follows: for the case of $\alpha=0.8$, we obtained $\tau=9.46 \mathrm{~h}, \varphi_{\mathrm{az}}=60.1^{\circ}$ and $d=0.88$; for the case of $\alpha=0.5$, we obtained $\tau=8.86 \mathrm{~h}$, $\varphi_{\mathrm{az}}=68.2^{\circ}$ and $d=0.92$; for the case of $\alpha=0.25$, we ob-

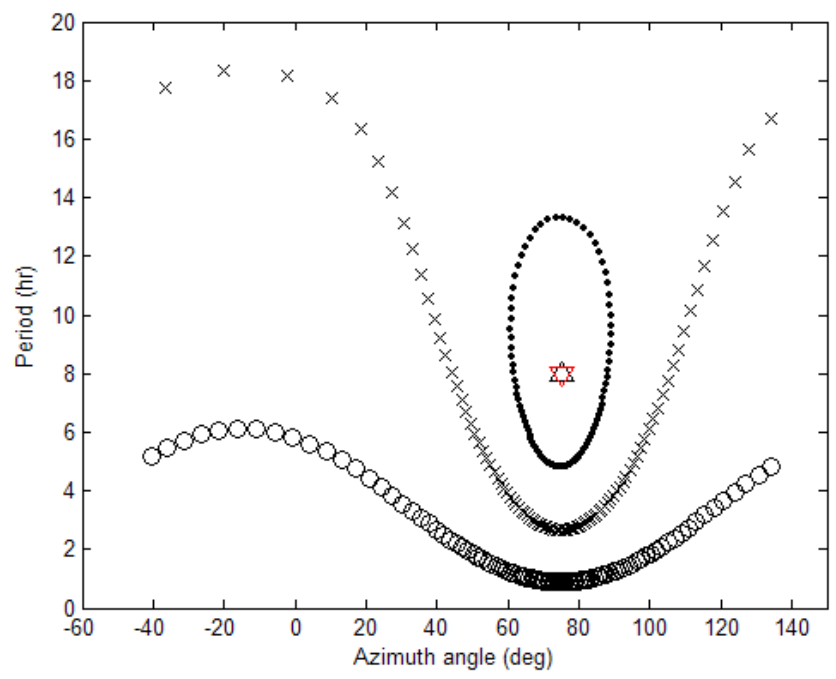

Fig. 3. Plot of period vs. azimuth angle resulted from hodograph analysis of Eqs. (8a), (8b), (8c) at each time step of three cases and the original waves. An open circle represents the results from the case with $\alpha=0.8$ in Eqs. (8a), (8b), (8c); a cross represents the results from the case with $\alpha=0.5$; and a dot represents the results from the case with $\alpha=0.25$. A black upward pointing triangle represents original wave with downward phase velocity (first wave in Table 1a) and a red downward pointing triangle represents the original wave with upward phase velocity (second wave in Table 1a).

tained $\tau=8.27 \mathrm{~h}, \varphi_{\mathrm{az}}=73.2^{\circ}$ and $d=0.97$. The results revealed that the superposition of two waves of the same period and wavelengths with opposite vertical sense of propagation and different azimuth tended to increase the characteristic wave period and decrease the degree of polarization. And the resulting azimuth was close (but not equal) to the weighting average of their azimuth angles. Here we must emphasize that when height averages on the Stokes parameters $I, D, P$, $Q$ in Eqs. (5a)-(5d) were not taken, the results of intrinsic period, azimuth angle and the degree of polarization at each height were varying in height with large fluctuations.

To investigate the effect of opposite vertical propagation, we reversed the vertical sense of propagation of the second wave in Table $1 \mathrm{~b}$ to make the phases of both waves propagating downward as shown in Table 1c. We generated data of three cases using Eqs. (8a), (8b), (8d) and Table $1 \mathrm{c}$ with $\alpha=0.8,0.5$ and 0.25 , respectively. Stokes parameters analyses of these three cases at each height (without doing height averages in Eqs. 5a, 5b, 5c, 5d) were height independent as follows: for the case of $\alpha=0.8$, we obtained $\tau=7.98 \mathrm{~h}, \varphi_{\mathrm{az}}=57.3^{\circ}$ and $d=1.0$; for the case of $\alpha=0.5$, we obtained $\tau=7.98 \mathrm{~h}, \varphi_{\mathrm{az}}=61.3^{\circ}$ and $d=1.0$; for the case of $\alpha=0.25$, we obtained $\tau=7.98 \mathrm{~h}, \varphi_{\mathrm{az}}=67.3^{\circ}$ and $d=1.0$. Unlike previous cases corresponding to Table $1 \mathrm{~b}$, these resulting periods were practically equal to the original waves and the resulting azimuths were equal to the weighting average of azimuths of the composition waves 


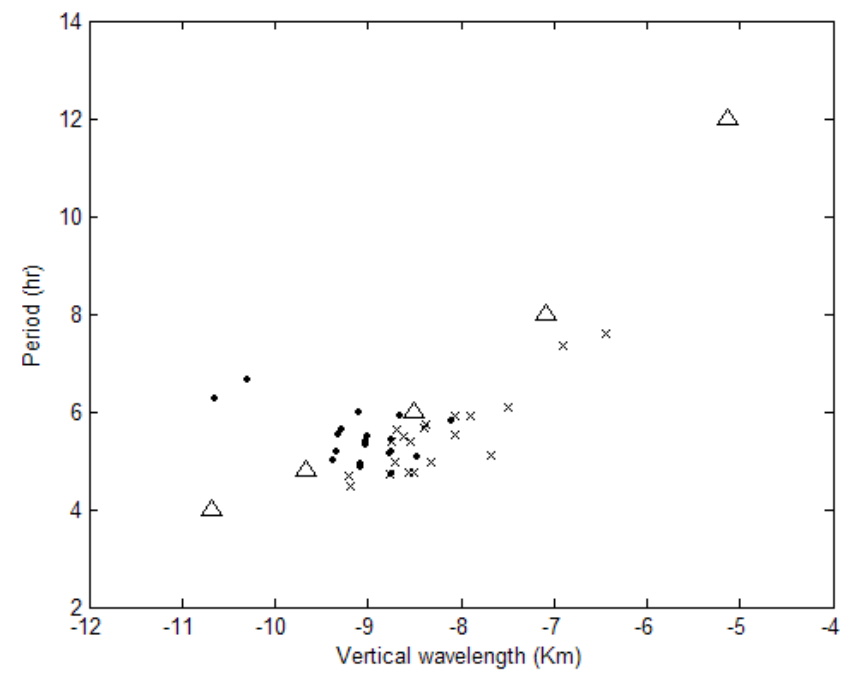

Fig. 4. Period vs. vertical wavelength plot of the component waves in Table 2a (by upward triangle) and the investigations of wave packets such as that in Fig. 7 by phase and group velocity tracing technique (by a cross). When all the component waves in Table $2 \mathrm{a}$ propagate in same direction with azimuth of 20 degrees (Table $2 c$ ), the velocity tracing investigations of the corresponding wave packets were presented by a "dot".

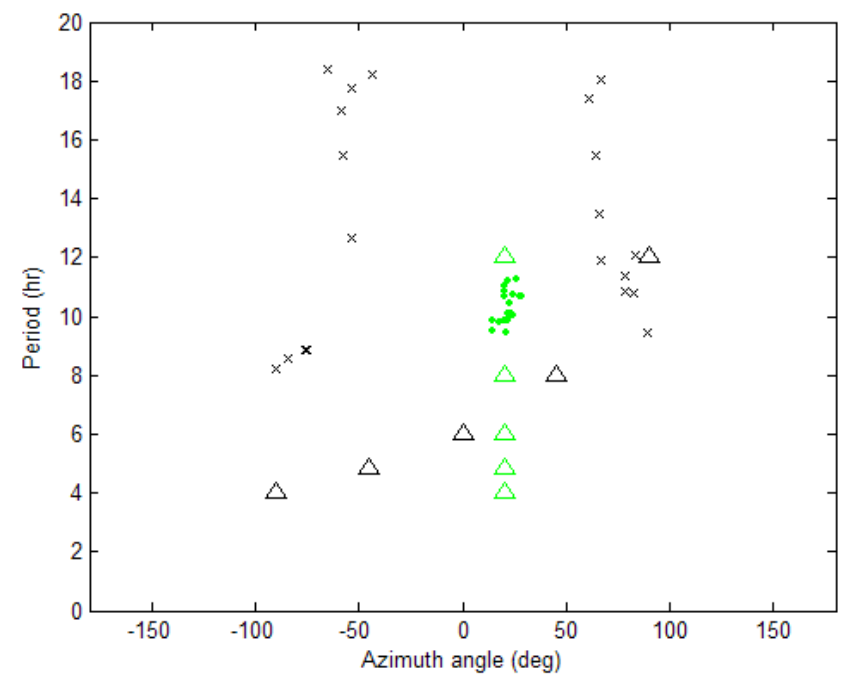

Fig. 5. Period vs. azimuth plot of the component waves in Table 2a (by a black upward triangle) and the results of hodograph analysis (by a black cross) around each wave packets as listed in Table 3. When all the component waves in Table 2 a propagate in same direction with azimuth of 20 degrees (see Table $2 \mathrm{c}$ ) as shown by a green upward triangle, the corresponding hodograph analyses were presented by a "green dot".

(i.e., $\left.\phi=\left(\phi_{1}+\alpha \phi_{2}\right) /(1+\alpha)\right)$. Almost the same results of wave period and azimuth were also obtained by hodograph analysis. So, it would be better if upward- and downwardpropagating waves were separately treated by Stokes param-
Table 2a. Properties of five upward propagating AGWs with $180^{\circ}$ azimuth spreading to be superposed.

\begin{tabular}{cccccc}
\hline$j$ & $\tau(\mathrm{h})$ & $\lambda_{\mathrm{h}}(\mathrm{km})$ & $\varphi_{\mathrm{az}}\left({ }^{\circ}\right)$ & $\lambda_{\mathrm{Z}}(\mathrm{km})$ & $A_{j}$ \\
\hline 1 & 12 & 900 & 90 & -5.136 & $A_{1}$ \\
2 & 8 & 734.8 & 45 & -7.095 & $A_{2}$ \\
3 & 6 & 636.4 & 0 & -8.501 & $A_{3}$ \\
4 & 4.8 & 569.2 & -45 & -9.666 & $A_{4}$ \\
5 & 4 & 519.6 & -90 & -10.689 & $A_{5}$ \\
\hline
\end{tabular}

Table 2b. Same as Table 2a, but for $90^{\circ}$ azimuth spreading only.

\begin{tabular}{cccccc}
\hline$j$ & $\tau(\mathrm{h})$ & $\lambda_{\mathrm{h}}(\mathrm{km})$ & $\varphi_{\mathrm{az}}\left({ }^{\circ}\right)$ & $\lambda_{\mathrm{z}}(\mathrm{km})$ & $A_{j}$ \\
\hline 1 & 12 & 900 & 90 & -5.136 & $A_{1}$ \\
2 & 8 & 734.8 & 67.5 & -7.095 & $A_{2}$ \\
3 & 6 & 636.4 & 45 & -8.501 & $A_{3}$ \\
4 & 4.8 & 569.2 & 22.5 & -9.666 & $A_{4}$ \\
5 & 4 & 519.6 & 0 & -10.689 & $A_{5}$ \\
\hline
\end{tabular}

Table 2c. Same as Table 2a, but for identical azimuth angles.

\begin{tabular}{cccccc}
\hline$j$ & $\tau(\mathrm{h})$ & $\lambda_{\mathrm{h}}(\mathrm{km})$ & $\varphi_{\mathrm{az}}\left({ }^{\circ}\right)$ & $\lambda_{\mathrm{z}}(\mathrm{km})$ & $A_{j}$ \\
\hline 1 & 12 & 900 & 20 & -5.136 & $A_{1}$ \\
2 & 8 & 734.8 & 20 & -7.095 & $A_{2}$ \\
3 & 6 & 636.4 & 20 & -8.501 & $A_{3}$ \\
4 & 4.8 & 569.2 & 20 & -9.666 & $A_{4}$ \\
5 & 4 & 519.6 & 20 & -10.689 & $A_{5}$ \\
\hline
\end{tabular}

eters analysis, but it is necessary that upward and downward waves must be separately treated by hodograph analysis.

\subsection{Superposition of five upward propagating waves}

Now let's consider the case of superposition of 5 downward phase velocity AGWs whose properties were listed in Table 2a, and indicated by black upward triangle in the period vs. vertical wavelength plot of Fig. 4 and in the period vs. azimuth plot of Fig. 5. Notice that each wave in Table 2a had different azimuth, period and wavelength, and its amplitude $A_{j}$ would be defined case by case. The perturbation velocity profiles obtained by Eqs. (7a), (7b), (7c), (7d) with $A_{1}=A_{2}=A_{3}=A_{4}=A_{5}=0.1$ (to be referred as Case M1 hereafter) were presented in Fig. 6a and b. The former one (Fig. 6a) presenting the time variations of perturbation velocities at successive heights, was prepared for phase and group velocity tracing analysis and Stokes parameters analysis; while the later (Fig. 6b) presenting the height variations of perturbation velocities at successive times, was prepared for hodograph analysis. 
Table 3. Results of wave packet analysis. Vertical wavelength $\lambda_{\mathrm{Z}}$ with negative sign indicates downward phase propagation. The star $(*)$ sign indicates the event satisfies dispersion equation under following condition: $\left|\left(v_{\mathrm{gz}}-\tilde{v}_{\mathrm{gz}}\right) / v_{\mathrm{gz}}\right|<0.15$ and $\left|\left(v_{\mathrm{pz}}-\tilde{v}_{\mathrm{pz}}\right) / v_{\mathrm{pz}}\right|<0.15$. EG and EP are, respectively, the percentage error of vertical group and phase velocities:

$\mathrm{EG}=\left|\left(v_{\mathrm{gz}}-\tilde{v}_{\mathrm{gz}}\right) / v_{\mathrm{gz}}\right| \times 100 \% \quad \mathrm{EP}=\left|\left(v_{\mathrm{pz}}-\tilde{v}_{\mathrm{pz}}\right) / v_{\mathrm{pz}}\right| \times 100 \%$

\begin{tabular}{cccccccc}
\hline $\mathrm{ID}$ & $v_{\mathrm{pz}}\left(\mathrm{m} \mathrm{s}^{-1}\right)$ & $v_{\mathrm{gz}}\left(\mathrm{m} \mathrm{s}^{-1}\right)$ & $\tau(\mathrm{h})$ & $\lambda_{\mathrm{z}}(\mathrm{km})$ & $\lambda_{\mathrm{h}}(\mathrm{km})$ & $\mathrm{EP}(\%)$ & $\mathrm{EG}(\%)$ \\
\hline $1^{*}$ & -0.489 & 0.481 & 4.95 & -8.71 & 534.6 & 1 & 4 \\
$2^{*}$ & -0.498 & 0.450 & 4.748 & -8.51 & 495.69 & 0 & 4 \\
$3^{*}$ & -0.518 & 0.515 & 4.705 & -8.77 & 508.14 & 0 & 5 \\
$4^{*}$ & -0.406 & 0.468 & 5.516 & -8.06 & 575.87 & 8 & 15 \\
$5^{*}$ & -0.500 & 0.508 & 4.762 & -8.57 & 497.15 & 3 & 5 \\
$6^{*}$ & -0.465 & 0.515 & 4.972 & -8.32 & 536.98 & 5 & 12 \\
$7^{*}$ & -0.570 & 0.482 & 4.478 & -9.19 & 483.62 & 4 & 7 \\
$8^{*}$ & -0.547 & 0.450 & 4.67 & -9.2 & 521.17 & 4 & 9 \\
$9^{*}$ & -0.440 & 0.462 & 5.393 & -8.54 & 582.44 & 5 & 7 \\
10 & -0.418 & 0.539 & 5.095 & -7.67 & 652.15 & 15 & 17 \\
$11 *$ & -0.412 & 0.498 & 5.661 & -8.4 & 662.63 & 12 & 15 \\
$12 *$ & -0.404 & 0.480 & 5.752 & -8.37 & 628.2 & 13 & 13 \\
$13 *$ & -0.429 & 0.424 & 5.629 & -8.69 & 592.27 & 3 & 4 \\
14 & -0.378 & 0.474 & 5.925 & -8.06 & 622.31 & 13 & 20 \\
$15 *$ & -0.436 & 0.451 & 5.484 & -8.61 & 592.14 & 5 & 7 \\
$16^{*}$ & -0.450 & 0.524 & 5.402 & -8.75 & 534.6 & 9 & 15 \\
17 & -0.371 & 0.462 & 5.909 & -7.89 & 495.69 & 15 & 16 \\
18 & -0.260 & 0.345 & 7.368 & -6.9 & 508.14 & 18 & 24 \\
19 & -0.235 & 0.342 & 7.61 & -6.44 & 575.87 & 24 & 29 \\
$20 *$ & -0.342 & 0.377 & 6.092 & -7.5 & 497.15 & 7 & 13 \\
\hline
\end{tabular}

\subsubsection{Phase and group velocity tracing analysis}

The vertical group velocity $v_{\mathrm{gz}}$, vertical phase velocity $v_{\mathrm{pz}}$, and the characteristic wave period $\tau$ of a wave packet were determined directly by the technique of phase and group velocity tracing (see Sect. III of Kuo et al., 2003) and the observed wave frequency $\sigma$ and vertical wave number $m$ were obtained readily by $\sigma=1 / \tau$ and $m=\sigma / v_{\mathrm{pz}}$, respectively.

A partial range-time plot of $(\delta V)^{2}$ derived from the left panel of Fig. 6a was shown in Fig. 7, where 10 wave packets were identified and determined by phase and group velocity tracing technique. A total number of 20 wave packets (10 from $u$-profile and 10 from $v$-profile) were investigated and the results of investigations were listed in Table 3 and presented by cross in Fig. 4. Among these 20 events, 15 of them (denoted by a star in Table 3) satisfied the dispersion equation under following condition:

$$
\left|\left(v_{\mathrm{gz}}-\tilde{v}_{\mathrm{gz}}\right) / v_{\mathrm{gz}}\right|<0.15 \text { and }\left|\left(v_{\mathrm{pz}}-\tilde{v}_{\mathrm{pz}}\right) / v_{\mathrm{pz}}\right|<0.15 \text {. }
$$

(Here $\tilde{v}_{\mathrm{gz}}$ and $\tilde{v}_{\mathrm{pz}}$ were theoretical values of vertical group and phase velocity best fitted by dispersion equation). Their mean values of the characteristic wave periods, vertical wavelengths and horizontal wavelengths were obtained to be $5.21 \mathrm{~h},-8.55 \mathrm{~km}$ and $549.5 \mathrm{~km}$ with small standard deviations of $0.49 \mathrm{~h}, 0.42 \mathrm{~km}$ and $53.6 \mathrm{~km}$, respectively. These mean values were closely associated with $j=3$ and $j=4$ wave (representing higher frequency part among the component waves) in Table $2 \mathrm{a}$. One should not expect these wave packet analyses to be perfect, because the wave packets in this study were formed from five discrete waves with different horizontal wavelengths. However, the theoretical vertical group velocity was defined by partial derivative of frequency with respect to vertical wave number at constant horizontal wave vector as follows,

$v_{\mathrm{gz}}=\frac{\partial \sigma}{\partial m}=\frac{\partial \omega}{\partial m}=\frac{-m\left(\omega^{2}-f^{2}\right)}{\omega\left(k^{2}+\ell^{2}+m^{2}+\frac{1}{4 H^{2}}\right)}$.

Apart from wave period, it was intuitively believed that the amplitude distribution of waves should also play an important role. So we changed the amplitudes of each component wave in Table 2a to: $A_{1}=0.1, A_{2}=0.08, A_{3}=0.06$, $A_{4}=0.05, A_{5}=0.04$ (to be referred as Case M2 hereafter), and $A_{1}=0.04, A_{2}=0.05, A_{3}=0.06, A_{4}=0.08, A_{5}=0.1$ (to be referred as Case M3 hereafter). Another factor worthy of studying is the azimuth distribution, so we reduced the azimuth spreading by replacing the azimuths of each component wave in Table $2 \mathrm{a}$ by azimuths in Table $2 \mathrm{~b}$, and generated three datasets using Eqs. (7a)-(7d) and Table $2 b$ with amplitudes defined as follows, Case M4: $A_{1}=A_{2}=A_{3}=$ $A_{4}=A_{5}=0.1 ;$ Case M5: $A_{1}=0.1, A_{2}=0.08, A_{3}=0.06$, $A_{4}=0.05, A_{5}=0.04$; Case M6: $A_{1}=0.04, A_{2}=0.05$, 

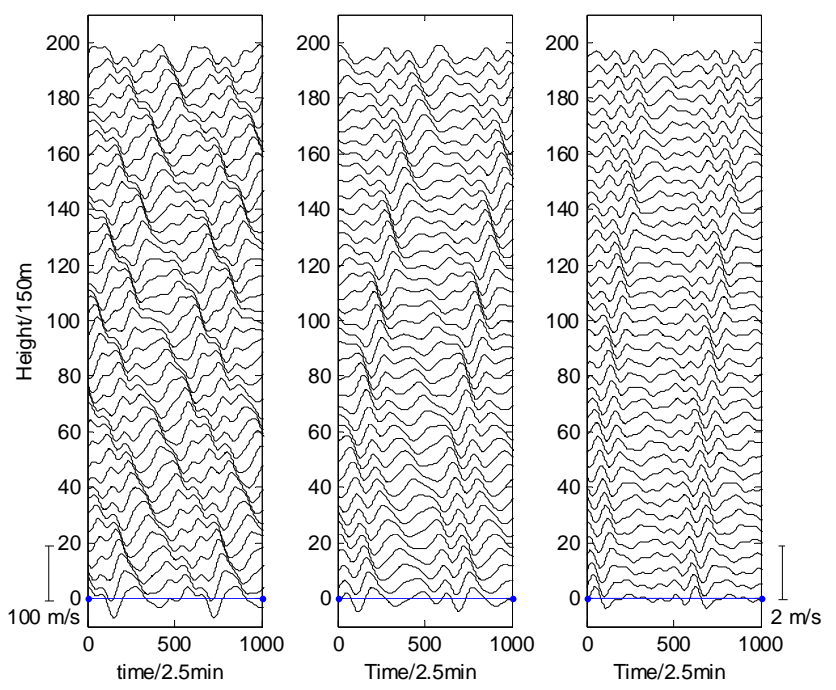

Fig. 6a. Time variations of perturbation velocities at successive heights. Left panel: zonal velocity profile; middle panel: meridional velocity profile; right panel: vertical velocity profile. The data were obtained from Eqs. (7a), (7b), (7c) and Table 2a with $\left(A_{1}, A_{2}, A_{3}, A_{4}, A_{5}\right)=(0.1,0.1,0.1,0.1,0.1)$. The time step is $2.5 \mathrm{~min}$ and the height resolution is $150 \mathrm{~m}$. The horizontal lines indicate $0 \mathrm{~m} \mathrm{~s}^{-1}$ for the first profile, and successive profiles are $0.75 \mathrm{~km}$ apart. The velocity scale of zonal and meridional velocities are indicated at the lower left corner of this figure, and the velocity scale of the vertical velocity is indicated at the lower right corner of this figure. These velocity profiles were prepared for wave packet analysis (phase and group velocity tracing technique) and Stokes parameters analysis.

$A_{3}=0.06, A_{4}=0.08, A_{5}=0.1$. In each case, 20 wave packets were investigated, and $n$ (case by case) out of the 20 wave packets satisfied condition (9). The results of cases M1M6 were summarized in Table 4, which revealed that the characteristic parameters of the wave packets were all dominated by the higher frequency wave components. It should be emphasized here that in windless situation, the azimuth of wave propagation cannot be determined from the dispersion Eq. (1b). If non-negligible background wind velocity is known, two azimuths symmetric with respect to the background wind velocity direction can be obtained from Eqs. (1a), (1b) and (10). And the true azimuth can be determined from these two symmetric azimuths with the help of momentum flux measurement (Kuo et al., 2009).

\subsubsection{Hodograph analysis}

To make comparison with the results from the phase and group velocity tracing technique, we made hodograph analysis around each wave packet (in terms of time and height range) in Table 3. The senses of rotations of all the hodographs were found to be clockwise, meaning downward phase velocity as expected. One example of hodograph (ID = 13 in Table 3) was shown in Fig. 8, whose major to
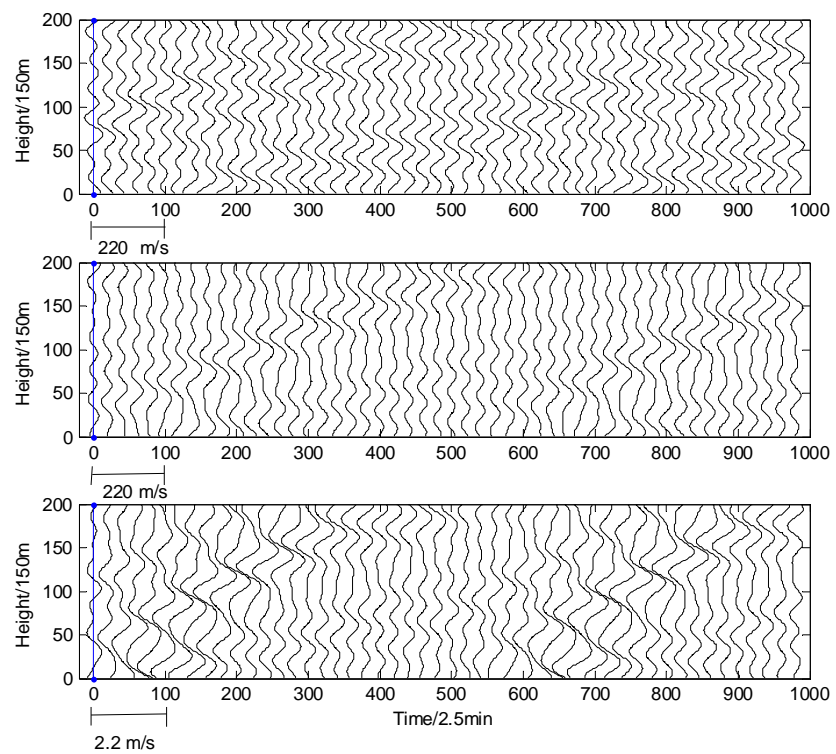

Fig. 6b. Height variations of perturbation velocities at successive times. Top panel: zonal velocity profile; middle panel: meridional velocity profile; bottom panel: vertical velocity profile. The time step is $2.5 \mathrm{~min}$ and the height resolution is $150 \mathrm{~m}$. The data were obtained from Eqs. (7a), (7b), (7c) and Table 2a with $\left(A_{1}, A_{2}, A_{3}, A_{4}, A_{5}\right)=(0.1,0.1,0.1,0.1,0.1)$. The vertical lines indicated $0 \mathrm{~m} \mathrm{~s}^{-1}$ for the first profile, and successive profiles are $50 \mathrm{~min}$ apart. The velocity scales are indicated at the bottom of each panel.. These velocity profiles were prepared for hodograph analysis.

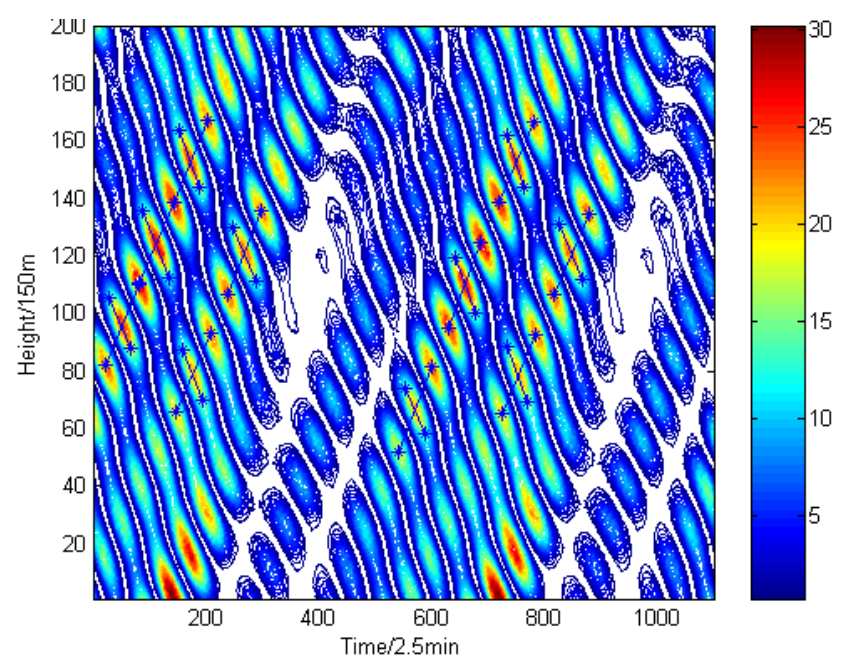

Fig. 7. A partial range-time plot of $(\delta V)^{2}$ of zonal velocity converted from left panel of Fig. 6a. Determinations of vertical phase and group velocities of wave packets were indicated by the phase lines (along the patch) and energy lines (across the patch).

minor axis ratio yields an intrinsic period of $15.45 \mathrm{~h}$, and its major axis lay along the direction with $\varphi_{\mathrm{az}}=63.3^{\circ}$. This intrinsic period is larger than each of the original wave in 


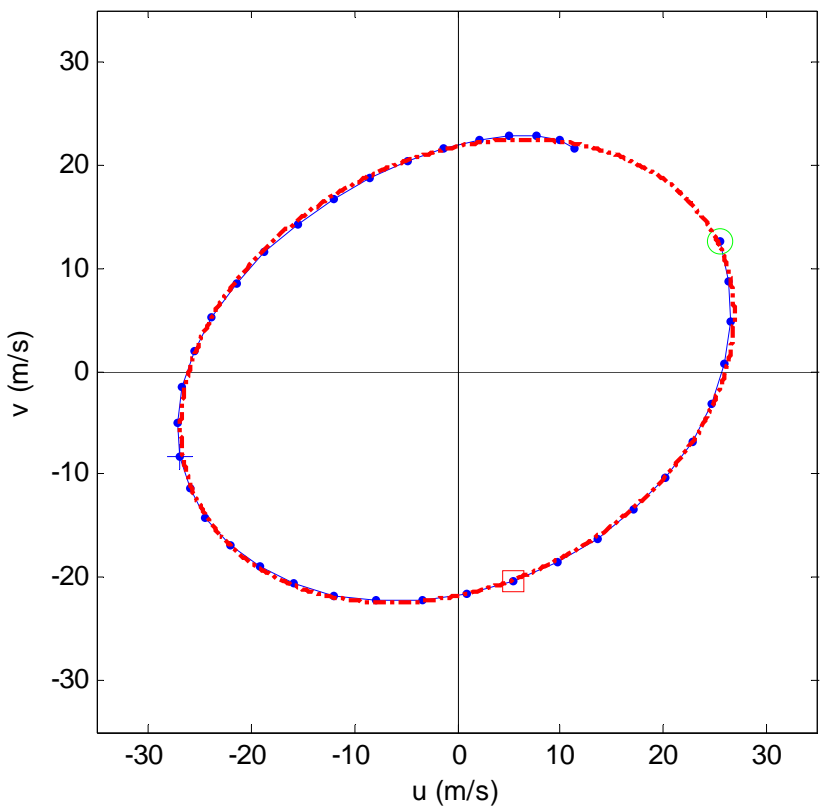

Fig. 8. Hodograph at $t=250 \Delta t$ and height range $105 \Delta z-141 \Delta \mathrm{z}$ of Fig. 6b. Least square fitting by an ellipse yields $\tau=15.45 \mathrm{~h}$ and $\varphi_{\mathrm{az}}=63.3^{\circ}$. The rotation sense of this hodograph is clockwise.

Table 4. Results of phase and group velocity analysis: the number of wave packets $n$ satisfying condition (9), and their mean \pm standard deviation of their intrinsic period $\tau(\mathrm{h})$, vertical wavelength $\lambda_{\mathrm{Z}}(\mathrm{km})$, and horizontal wave length $\lambda_{\mathrm{h}}(\mathrm{km})$.

\begin{tabular}{ccccc}
\hline & $n$ & $\tau$ & $\lambda_{\mathrm{z}}$ & $\lambda_{\mathrm{h}}$ \\
\hline Case M1 & 15 & $5.21 \pm 0.49$ & $-8.55 \pm 0.42$ & $549.5 \pm 53.6$ \\
Case M2 & $15^{\mathrm{a}}$ & $6.18 \pm 0.49$ & $-8.61 \pm 0.38$ & $665.8 \pm 53.6$ \\
Case M3 & 14 & $4.91 \pm 0.52$ & $-9.16 \pm 0.30$ & $553.7 \pm 64.3$ \\
Case M4 & 12 & $5.19 \pm 0.57$ & $-8.90 \pm 0.53$ & $567.2 \pm 47.8$ \\
Case M5 & 10 & $5.18 \pm 0.28$ & $-8.61 \pm 0.28$ & $550.4 \pm 45.8$ \\
Case M6 & 17 & $4.89 \pm 0.66$ & $-9.22 \pm 0.44$ & $552.7 \pm 73.6$ \\
\hline
\end{tabular}

a Actually, there were only 6 wave packets satisfying Eq. (9) in Case M2, so we relaxed the condition from 0.15 to 0.20 in Eq. (9) to increase the number of wave packets.

Table 2a. The results of hodograph analysis for all the wave packets were listed in Table 5 and presented by a "cross" in the period vs. azimuth plot in Fig. 5, which revealed that half of the hodographs yielded wave periods well beyond the spectrum of the input waves in Table $2 \mathrm{a}$. We also noticed that the hodograph in Fig. 8 was not complete, because those data points that deviated away from the ellipse were removed from the dataset and the remaining data were refitted again by an ellipse. Such fitting process was repeated until best fitting was reached. Actually, none of the hodographs in this case was complete. So we did not proceed to make hodograph analysis for Cases M2-M6.
Table 5. Results of hodograph analysis corresponding to each wave packet in Table 3.

\begin{tabular}{rrrrr}
\hline ID & $\begin{array}{r}\text { Major axis } \\
\mathrm{a}\left(\mathrm{m} \mathrm{s}^{-1}\right)\end{array}$ & $\begin{array}{r}\text { Minor axis } \\
\mathrm{b}\left(\mathrm{m} \mathrm{s}^{-1}\right)\end{array}$ & $\tau(\mathrm{h})$ & $\varphi_{\mathrm{az}}\left(^{\circ}\right)$ \\
\hline 1 & 22.039 & 9.006 & 8.581 & -84.3 \\
2 & 18.849 & 7.962 & 8.871 & -76.3 \\
3 & 26.304 & 22.786 & 18.191 & -43.9 \\
4 & 30.445 & 24.622 & 16.984 & 58.1 \\
5 & 26.630 & 12.006 & 9.467 & 89.5 \\
6 & 23.392 & 9.174 & 8.236 & -89.8 \\
7 & 19.066 & 8.020 & 8.834 & -75.4 \\
8 & 27.328 & 23.072 & 17.73 & -53.4 \\
9 & 30.699 & 25.395 & 17.372 & 60.7 \\
10 & 23.990 & 12.335 & 10.797 & 82.3 \\
11 & 21.514 & 12.989 & 12.679 & -53.5 \\
12 & 24.085 & 21.100 & 18.398 & -65.0 \\
13 & 28.066 & 20.673 & 15.451 & 63.3 \\
14 & 30.092 & 17.056 & 11.903 & 66.7 \\
15 & 22.906 & 16.856 & 15.453 & -57.3 \\
16 & 25.669 & 22.061 & 18.048 & 66.9 \\
17 & 29.422 & 18.878 & 13.474 & 66.0 \\
18 & 23.314 & 12.022 & 10.829 & 78.6 \\
19 & 24.896 & 13.481 & 11.371 & 78.4 \\
20 & 16.775 & 9.665 & 12.099 & 83.0 \\
\hline
\end{tabular}

Table 6. Results of Stokes parameters analysis: intrinsic period, $\tau$ (h), $\varphi_{\mathrm{az}}\left({ }^{\circ}\right)$, and degree of polarization $d$.

\begin{tabular}{cccc}
\hline & $\tau$ & $\varphi_{\mathrm{az}}$ & $d$ \\
\hline Case M1 & 14.48 & 80.9 & 0.80 \\
Case M2 & 13.29 & 82.6 & 0.89 \\
Case M3 & 14.89 & 88.7 & 0.69 \\
Case M4 & 12.50 & 74.0 & 0.85 \\
Case M5 & 12.02 & 82.3 & 0.94 \\
Case M6 & 13.29 & 51.3 & 0.72 \\
\hline
\end{tabular}

\subsubsection{Stokes parameters analysis}

The result of Stokes parameters analyses of Cases M1-M6 were all height independent and the results of intrinsic period $\tau(\mathrm{h})$, azimuth angle $\varphi_{\mathrm{az}}\left(^{\circ}\right)$ and degree of polarization $d$ at each height were summarized in Table 6. The results of Case M2 and Case M3 were contradictory: The azimuth of the largest amplitude wave in Case M3 was $-90^{\circ}$, while the azimuth of the largest amplitude wave in Case M2 was $90^{\circ}$, yet the resulting azimuth of Case M3 was even closer than the resulting azimuth of Case M2 to $90^{\circ}$. These problematic results were apparently due to the fact that Stokes parameters method could not distinguish between azimuths $\phi_{\mathrm{az}}$ and $\phi_{\mathrm{az}} \pm 180^{\circ}$, therefore, it could not distinguish between $90^{\circ}$ and $-90^{\circ}$. When the azimuth spreading was reduced from $180^{\circ}$ in Table 2a (Cases M1-M3) to $90^{\circ}$ in Table $2 \mathrm{~b}$ 
(Cases M4-M6), the results were reasonable and we could conclude that both the resulting period and azimuth were dominated by the largest period wave. The fact that the resulting intrinsic periods were all larger than the longest period ( $12 \mathrm{~h}$ ) among the component waves was believed to be resulted from the azimuth spreading among the component waves.

\subsection{Superposition of five waves propagating in same direction vertically and horizontally}

A special case related to the previous cases was the superposition of five waves with same amplitude $\left(A_{1}=A_{2}=A_{3}=\right.$ $\left.A_{4}=A_{5}=0.1\right)$ and propagating in the same direction with $\varphi_{\mathrm{az}}=20^{\circ}$ as shown in Table 2c. These five waves were represented by a green vertical triangle in Fig. 5. Twenty wave packets were investigated by phase and group velocity tracing technique; the results of the investigations were similar to that of the previous case and were shown by a dot in period vs. vertical wavelength plot in Fig. 4 for comparison. Among these 20 events, 13 of them satisfy the dispersion equation under the condition of Eq. (9). Their mean values of the characteristic wave periods, vertical wavelengths and horizontal wavelengths were obtained to be $5.20 \mathrm{~h},-8.96 \mathrm{~km}$ and $569.16 \mathrm{~km}$ with small standard deviations of $0.34 \mathrm{~h}, 0.25 \mathrm{~km}$ and $33.45 \mathrm{~km}$, respectively. These mean values were closely associated with the wave of $j=3$ and $j=4$ in Table 2c. So, both case studies (Sects. 3.3.1 and 3.4) revealed that the results of wave packet analysis were closely associated with the high frequency part of the wave spectrum in Table 2c, because high frequency waves had better relative frequency resolution $\Delta \omega / \omega$ than low frequency waves (Kuo et al., 2003, 2009). The results of hodograph analysis associated with these 20 wave packets were shown by a green dot in Fig. 5, where the majority of the hodograph investigations fell in a region between the corresponding properties of $j=1$ and $j=2$ waves in Table $2 \mathrm{c}$, the mean value of their periods was $10.29 \mathrm{~h}$ with a standard deviation of $0.54 \mathrm{~h}$, and the mean value of their azimuth angles was $21.75^{\circ}$ with a standard deviation of $3.49^{\circ}$. The azimuth obtained by hodograph analysis yielded the same azimuth of the original waves $\left(20^{\circ}\right)$, and the corresponding wave period (around $10.29 \mathrm{~h}$ ) was associated with the lowest frequency part of the wave spectrum $(12 \mathrm{~h})$ in Table 2c. Finally, the analysis of this case by Stokes parameters method was: $\tau=9.86 \mathrm{~h}, \varphi_{\mathrm{az}}=20^{\circ}$ and $d=0.98$, which is qualitatively consistent with the statistical result of hodograph analysis.

\section{On Stokes parameters/rotary spectra method and technique of phase and group velocity tracing}

In the practical analysis of Stokes parameters, computing the circular polarization parameter $Q$ from Eq. (5d) is not straight forward because $\tilde{u}$ is not a measured quantity, which involves a $90^{\circ}$ phase shift from zonal fluctuation velocity $u$. Therefore, Eckermann and Vincent (1989) developed a spectra method for Stokes parameters analysis:

$u(z)=\operatorname{Re}\left\{\sum_{m}\left[U_{\mathrm{R}}(m)+i U_{\mathrm{I}}(m)\right] \cdot e^{i m z}\right\}$,

$v(z)=\operatorname{Re}\left\{\sum_{m}\left[V_{\mathrm{R}}(m)+i V_{\mathrm{I}}(m)\right] \cdot e^{i m z}\right\}$,

$\bar{I}=A \sum_{m}\left[\overline{U_{\mathrm{R}}^{2}(m)}+\overline{U_{\mathrm{I}}^{2}(m)}+\overline{V_{\mathrm{R}}^{2}(m)}+\overline{V_{\mathrm{I}}^{2}(m)}\right]$,

$\bar{D}=A \sum_{m}\left[\overline{U_{\mathrm{R}}^{2}(m)}+\overline{U_{\mathrm{I}}^{2}(m)}-\overline{V_{\mathrm{R}}^{2}(m)}-\overline{V_{\mathrm{I}}^{2}(m)}\right]$

$\bar{P}=2 A \sum_{m}\left[\overline{U_{\mathrm{R}}(m) V_{\mathrm{R}}(m)}+\overline{U_{\mathrm{I}}(m) V_{\mathrm{I}}(m)}\right]$,

$\bar{Q}=2 A \sum_{m}\left[\overline{U_{\mathrm{R}}(m) V_{\mathrm{I}}(m)}-\overline{U_{\mathrm{I}}(m) V_{\mathrm{R}}(m)}\right]$.

Here $A$ is a constant, "Re" denotes the real part of the complex number, subscriptions "R" and " $\mathrm{I}$ " of the complex amplitude $U$ and $V$ denote, respectively, the real part and imaginary part of the corresponding complex amplitude $U$ and $V$. Overbar represents time average. The range of time for time average and the range of summation of vertical wave number $m$ are to be properly selected to estimate the characteristic intrinsic frequency and azimuth as well as the degree of polarization of the wave packet. To separate clockwiserotating waves from anti-clockwise-rotating waves, a closely related method called rotary spectra method has been applied in oceanic and atmospheric studies (see Eckermann, 1996, and references therein). Its formulas were as follows,

$$
\begin{aligned}
u(z)+i v(z)= & \sum_{m}\left[\frac{U_{\mathrm{R}}(m)-V_{\mathrm{I}}(m)}{2}+i \frac{V_{\mathrm{R}}(m)+U_{\mathrm{I}}(m)}{2}\right] \\
& \cdot e^{i m z} \\
& +\sum_{m}\left[\frac{U_{\mathrm{R}}(m)+V_{\mathrm{I}}(m)}{2}+i \frac{V_{\mathrm{R}}(m)-U_{\mathrm{I}}(m)}{2}\right] \\
& \cdot e^{-i m z} .
\end{aligned}
$$

The coefficient of $e^{i m z}\left(e^{-i m z}\right)$ had been regarded as the complex amplitude of an anti-clockwise rotating (clockwiserotating) wave.

Equation (13) is nothing but a linear combination of Eqs. (11a) and (11b) in a complex form. We notice that Eqs. (11a), (11b) and (13) involve single Fourier transform over height assuming both perturbation velocities $u(z, t)$ and $v(z, t)$ have a time variation with a form of $e^{i \sigma t}$ instead of a combination of $e^{i \sigma t}$ and $e^{-i \sigma t}$ ( $\sigma$ and $m$ are positively defined). Consequently, these equations do not separate phaseupward from phase-downward propagating waves. This can 
be understood from following the example of wave superposition illustrated by Eqs. (14a), (14b): Assume the perturbation velocity $u(z, t)$ (so is $v(z, t)$ ) results from the superposition of phase-upward propagating waves (1st term of r.h.s. of Eq. 14a) and phase-downward propagating waves (2nd term of r.h.s. of Eq. 14a),

$$
\begin{aligned}
u(z, t)= & \sum_{\sigma, m}\{\alpha \cos (\sigma t-m z)+\beta \sin (\sigma t-m z)\} \\
& +\sum_{\sigma, m}\{\gamma \cos (\sigma t+m z)+\zeta \sin (\sigma t+m z)\} \\
= & \sum_{\sigma, m}\left\{\left(\frac{\alpha-i \beta}{2}\right) e^{i \sigma t}+\left(\frac{\gamma+i \zeta}{2}\right) e^{-i \sigma t}\right\} e^{-i m z} \\
& +\sum_{\sigma, m}\left\{\left(\frac{\gamma-i \zeta}{2}\right) e^{i \sigma t}+\left(\frac{\alpha+i \beta}{2}\right) e^{-i \sigma t}\right\} e^{i m z},
\end{aligned}
$$

where coefficients $\alpha, \beta, \gamma$ and $\zeta$ are real functions of $\sigma$ and $m$. Evidently, the complex coefficients of $e^{-i \sigma t}$ in Eq. (14b) cannot vanish simultaneously, therefore, it is impossible to separate upward waves from downward waves by single Fourier transform over height (or time) as rotary spectra method did.

In contrast to the rotary spectra method, the separation of upward and downward waves in phase and group velocity tracing technique involves double Fourier transform over height and time (see Sect. III-1 of Kuo et al., 2003). It does completely separate phase-upward propagating waves from phase-downward propagating waves, but does only partially separate waves with upward group velocity and waves with downward group velocity due to the Doppler effect. If the background wind is negligible, the intrinsic frequency $\omega$ will be equal to the observed frequency $\sigma$ ( $\sigma$ and $m$ are positively defined in Eq. 14b), then the group velocities and phase velocities of all the wave packets will have opposite sense of vertical propagation (to be referred as type 1 wave packets). If the background wind is not negligible, some waves may be Doppler shifted into negative intrinsic frequency (see Eq. 1a) causing their vertical group velocities and phase velocities to have the same sense of vertical propagation, and we called such wave packets as type 2 wave packets (Kuo et al., 2003, 2008, 2009; Kuo and Röttger, 2005). Phase and group velocity tracing technique can unambiguously identify the location (height and time) of type 1 and type 2 wave packets. Among previous wave packet researches, about $85 \%$ were type 1 while $15 \%$ were type 2 wave packets in one study of SOUSY-Svalbard Radar observation (Kuo et al., 2003); and about $76 \%$ were type 1 while $24 \%$ were type 2 wave packets in another study of SOUSY-Svalbard Radar observation (Kuo and Röttger, 2005). In a recent study of the MU Radar observation (Kuo et al., 2008), only $6 \%$ were type 2 wave packets and $94 \%$ were type 1 packets. These statistics revealed that type 2 wave packets represented approximately less than $25 \%$ of the gravity wave packets.

Phase and group velocity tracing technique offers three determinations: observed wave period, vertical phase velocity

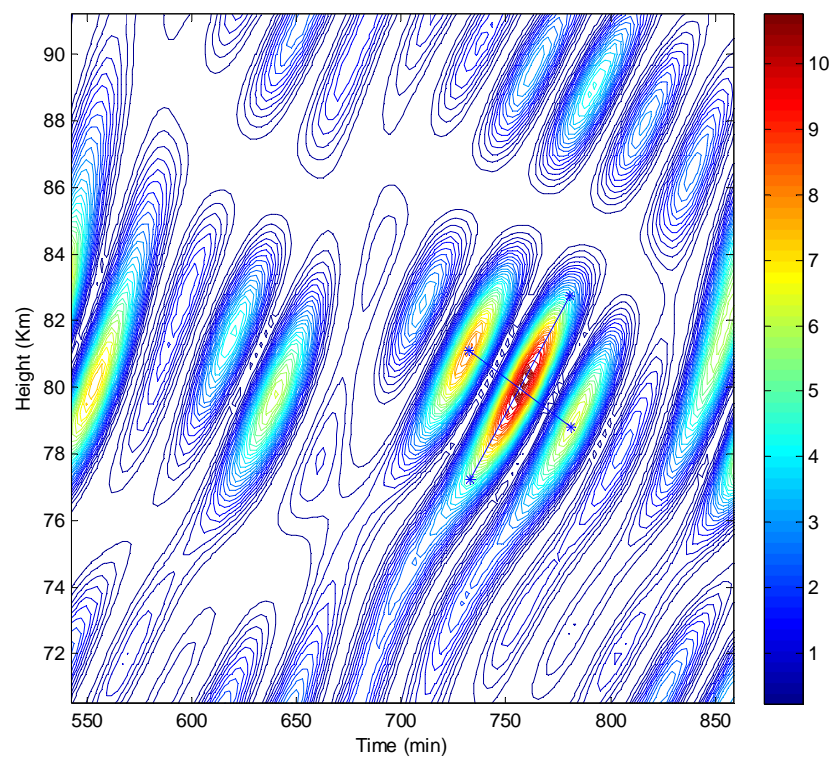

Fig. 9. A partial range-time plot of $(\delta V)^{2}$ of meridional velocity converted from dataset observed by the MU radar at Shigaraki Japan on 15 November 1988. Determination of vertical phase and group velocities of wave packets were indicated by the phase lines (along the patch) and energy lines (across the patch).

and vertical group velocity. From these three determinations observed frequency $\sigma$, vertical wavelength $\lambda_{\mathrm{z}}$, along with vertical group velocity $v_{\mathrm{gz}}$ are readily obtained. Then the intrinsic frequency $\omega$, horizontal wavelength $\lambda_{\mathrm{h}}$ and azimuth $\phi_{\mathrm{az}}$ (if the background wind velocity is known) can be obtained by fitting the three determinations into Doppler relation and dispersion Eqs. (1a), (1b) and its related Eq. (10) for vertical group velocity. In such a manner, the derived quantities $\omega, \lambda_{\mathrm{h}}$ and azimuth $\phi_{\mathrm{az}}$ were forced to satisfy Doppler relation and dispersion Eqs. (1a), (1b) and its related Eq. (10), but were not forced to satisfy polarization relation. By contrast, Stokes parameters/rotary spectrum method also offers three determinations following polarization relation: $\omega, \lambda_{\mathrm{z}}$ and $\phi_{\mathrm{az}}$. Then $\lambda_{\mathrm{h}}$ can be obtained from dispersion Eq. (1b). However, these parameters were not forced to satisfy Eqs. (1a) and (10). Evidently, these two methods are complementary to each other, and it may be worth developing a composite method of wave packet analysis combining these two methods.

\section{An example of composite wave packet analysis of real radar data}

Let us have a look at one example of wave packet analysis as shown in Fig. 9, which was a partial range-time plot of $(\delta V)^{2}$ of meridional fluctuation velocity obtained from a mesospheric data observed by the $\mathrm{MU}$ radar $\left(35^{\circ} \mathrm{N}, 136^{\circ} \mathrm{E}\right)$ at Shigaraki Japan, on 17 November 1988. The time range of 
this dataset was 9 AM-3 PM with time resolution of $147 \mathrm{~s}$; and the height range was $63.6-99.3 \mathrm{~km}$ with height resolution of $300 \mathrm{~m}$. The constant mean wind velocity had a magnitude of $30.97 \mathrm{~m} \mathrm{~s}^{-1}$ with an azimuth angle of $65.54^{\circ}$. A time window of $30 \mathrm{~min}-3 \mathrm{~h}$ (2nd to 12 th frequency mode) and wavelength window of $5.95 \mathrm{~km}$ to $35.7 \mathrm{~km}$ (1st to 6th wave number mode) to separate upward phase velocity waves from downward phase velocity waves.

The vertical phase velocity, vertical group velocity and characteristic wave period of the wave packet in Fig. 9 determined by phase and group velocity tracing technique were $\tau=69 \mathrm{~min}, v_{\mathrm{pz}}=1.917 \mathrm{~m} \mathrm{~s}^{-1}$ and $v_{\mathrm{gz}}=-0.831 \mathrm{~m} \mathrm{~s}^{-1}$, respectively. The vertical wavelength $\lambda_{\mathrm{z}}=7.94 \mathrm{~km}$ was readily obtained. Substituting these values into Doppler relation and dispersion equations and its derived group velocity equations, we obtained the characteristic intrinsic period, horizontal wavelength and azimuth angle as follows: $\tau=2.51 \mathrm{~h}$, $\lambda_{\mathrm{h}}=236.1 \mathrm{~km}$ and $\phi_{\mathrm{az}}=60.9^{\circ}$ or $69.9^{\circ}$, with relative error of vertical group velocity $\left|\left(v_{\mathrm{gz}}-\tilde{v}_{\mathrm{gz}}\right) / v_{\mathrm{gz}}\right|=0.01$ and phase velocity $\left|\left(v_{\mathrm{pz}}-\tilde{v}_{\mathrm{pz}}\right) / v_{\mathrm{pz}}\right|=0.01$, satisfying condition (9).

The uncertainty of azimuth angles $\left(\phi_{\mathrm{az}}=60.9^{\circ}\right.$ or $\left.69.9^{\circ}\right)$ can be solved with the help of Stokes parameters analysis. First of all, we noticed that phase and group velocity technique tends to yield wave parameters corresponding to high frequency part of the wave packet's component waves, while Stokes parameters analysis tends to yield a result corresponding to a low frequency part of the wave packet's component waves. So we use a time window of $30 \mathrm{~min}$ to $1 \mathrm{~h}$ (6th to 12th frequency mode) and wavelength window of $5.95 \mathrm{~km}$ to $35.7 \mathrm{~km}$ (1st to 6 th wave number mode, same as the window for velocity tracing analysis) to separate upward phase velocity waves from downward phase velocity waves. Then we applied spectra method of Stokes parameters analysis to calculate Stokes parameters from Eqs. (12a)-(12d) using the 4 th wave number mode ( $m=4$, corresponding to $8.925 \mathrm{~km}$ of vertical wavelength), and taking time average over a time range of $138 \mathrm{~min}$ (corresponding to 2 periods time of the wave packet) with its time centre right at the time centre of the wave packet $(756.5 \mathrm{~min})$ in Fig. 9. We finally obtained intrinsic wave period $\tau=1.69 \mathrm{hr}$ and azimuth angle $\phi_{\mathrm{az}}=74.1^{\circ}$ or $\phi_{\mathrm{az}}=254.1^{\circ}$. Comparing with the result of phase and group velocity tracing technique $(\tau=2.51 \mathrm{~h}$, $\lambda_{\mathrm{h}}=236.1 \mathrm{~km}$ and $\phi_{\mathrm{az}}=60.9^{\circ}$ or $69.9^{\circ}$ ), the intrinsic periods were reasonably close to each other and we decided that the azimuth of the wave packet was $69.9^{\circ}$. So the projection horizontal wavelength along the north-south line was $687 \mathrm{~km}$, which was long enough to make the error in meridional wind measurement (by dual beam method) negligible. Here we would like to emphasize that we had used different combinations of the range of vertical wave numbers for mode summation and the range of time for time average to calculate Stokes parameters, and the result above was closest to the result of velocity tracing technique. Detailed results of the study of mesospheric data observed by the MU radar will be presented in a separate paper (Kuo et al., 2012).

\section{Summary}

We may briefly summarize the results of the simulation studies as follows. For the case of one monochromatic wave, hodograph analysis was perfect to obtain both the wave period and its propagation direction; Stokes parameters method was accurate to estimate the period and azimuth irrespective of its vertical sense of propagation, but could not distinguish between azimuths $\phi_{\mathrm{az}}$ and $\phi_{\mathrm{az}} \pm 180^{\circ}$. The $180^{\circ}$. ambiguity can be solved by correlation with simultaneous measurements of temperature oscillations due to the polarization between temperature, zonal and meridional wind. In the case of two waves with same wave periods and wavelengths, but different amplitudes propagating in opposite vertical direction, the hodograph would be perfectly fitted by an ellipse and revealed the same sense of vertical propagation of the major wave even when the amplitude ratio of minor to major wave was as large as 0.8. But the resulting wave period and azimuth from hodograph analysis was unacceptably erratic even when the amplitude ratio of minor to major wave was as small as 0.25 . Stokes parameters method would yield the wave period larger than the original period, and the resulting azimuth was close (but not equal) to the weighting average of their azimuth angles. So separation of data into sets of upward propagating waves and downward propagating waves before doing analysis is essential for hodograph analysis. Then we studied the simulation data in which all component waves propagated in the same vertical direction. For the case of superposition of 5 waves of different periods, wavelengths and azimuths as listed in Table 2a, the phase and group velocity tracing technique yielded that characteristic wave period, vertical wavelength and horizontal wavelength were all closely associated with the higher frequency part among the component waves. Though hodograph analysis failed to yield reasonable result, its counterpart, Stokes parameters method, did yield reasonable period and azimuth if the spreading in azimuths of all component waves did not exceed $90^{\circ}$, or more specifically, the resulting period would be larger than the period of the lowest frequency component wave, and the resulting azimuth would be dominated by the lowest frequency component wave. If all five waves in Table 2 a propagated in the same direction vertically and horizontally, then all the three methods were meaningful: The resulting azimuth from hodograph method and Stokes parameters method were consistent with the original waves, and their resulting periods tended to correspond to the low frequency part among the component waves, while the result from the phase and group velocity tracing technique tended to correspond to high frequency part among the component waves.

As a conclusion, we suggest that separation of upward propagating waves from downward propagating waves has to be made, and upward data and downward data must be treated independently. Then, Stokes parameters method would yield the characteristic intrinsic wave period, vertical 
wavelength and azimuth angle following polarization relation; phase and group velocity tracing would identify the locations of wave packets and determine their characteristic wave period, vertical phase and group velocities, then horizontal wavelength and azimuth could be estimated from dispersion equation and its related formula for vertical group velocity. However, if the continuous data record is too short, up-down separation process (see Sect. III-1 of Kuo et al., 2003) might give rise to serious error due to border effect and poor frequency resolution, then phase and group velocity tracing technique might be less credible.

In case upward waves and downward waves cannot be effectively separated, Stokes parameters method may remain the only effective method in determining gravity wave parameters due to its insensitivity to the vertical sense of propagation. Conventionally, wavelet analysis was applied to identify the location and the dominant scale of wave event, then narrow band filter was applied to pick out a specific signal for hodograph analysis and Stokes parameters analysis (Sato and Yamada, 1994; Serafimovich et al., 2005; Hoffmann et al., 2006; Chagnon and Gray, 2008).

For a gravity wave packet, phase and group velocity tracing technique will yield characteristic wave parameters corresponding to the high frequency part of the wave packet; while Stokes parameters method will give characteristic wave parameters corresponding to the low frequency part. Phase and group velocity tracing technique follows dispersion equation and its related formula, while spectra method of Stokes parameters analysis follows polarization relation. It is fair to say that these two methods are complementary to each other. However, the existence of type 2 wave packets propagating in the wind field would cause some trouble in measuring upward/downward ratio of energy transported by gravity wave. Phase and group velocity tracing is able to identify their locations for proper treatment, while Stokes parameters method does not have this merit. Phase and group velocity tracing technique and dispersion equation would yield two azimuth angles symmetric with respect to the mean wind direction. This ambiguity of azimuth can be solved with the help of the spectra method of Stokes parameters analysis as demonstrated in this paper.

Acknowledgements. This work is supported in part by the National Science Council of Taiwan under the contract number NSC 992111-M-238-001. We deeply thank the anonymous referees for their invaluable comments on this manuscript.

Topical Editor C. Jacobi thanks three anonymous referees for their help in evaluating this paper.

\section{References}

Chagnon, J. M., and Gray, S. L.: Analysis of convectively-generated gravity waves in mesoscale model simulations and wind-profiler observations, Q. J. Roy. Meteorol. Soc. 134, 663-676, 2008.

Eckermann, S. D.: Hodographic analysis of gravity waves: Relationships among Stokes parameters, rotary spectra and crossspectral methods, J. Geophys. Res., 101, 19169-19174, 1996.

Eckermann, S. D. and Vincent, R. A.: Falling sphere observations of anisotropic gravity wave motions in the upper stratosphere over Australia, Pure Appl. Geophys., 130, 509-532, 1989.

Fritts, D. C. and Alexander, M. J.: Gravity wave dynamics and effects in the middle atmosphere, Rev. Geophys., 41, 1003, doi:10.1029/2001RG000106, 2003.

Gossard, E. E. and Hooke, W. H.: Waves in the Atmosphere, Elsevier, New York, p. 98, 1975.

Hamilton, K.: Climatological statistics of stratospheric inertiagravity waves deduced from historical rocketsonde wind and temperature data, J. Geophys. Res., 96, 20831-20839, 1991.

Hirota, I. and Niki, T.: A statistical study of inertial-gravity waves in the middle atmosphere, J. Meteorol. Soc. Jpn., 63, 1055-1066, 1985.

Hoffmann, P., Serafimovich, A., Peters, D., Dalin, P., Goldberg, R., and Latteck, R.: Inertia gravity waves in the upper troposphere during the MaCWAVE winter campaign - Part I: Observations with collocated radars, Ann. Geophys., 24, 2851-2862, doi:10.5194/angeo-24-2851-2006, 2006.

Kitamura, Y. and Hirota, I.: Small-scale disturbances in the lower stratosphere revealed by daily rawin sonde observations, J. Meteorol. SOC. Japan, 67, 817-831, 1989.

Kuo, F. S. and Röttger, J.: Horizontal wavelength of gravity wave in the lower atmosphere measured by the SOUSY Svalbard Radar, Chinese Journal of Physics, 43, 464-480, 2005.

Kuo, F. S., Röttger, J., and Lue, H. Y.: Propagation of gravity wave packets in the lower atmosphere observed by the SOUSYSvalbard radar, Chinese Journal of Physics, 41, 309-325, 2003.

Kuo, F. S., Lue, H. Y., Fern, C. L., Röttger, J., Fukao, S., and Yamamoto, M.: Studies of vertical fluxes of horizontal momentum in the lower atmosphere using the MU-radar, Ann. Geophys., 26, 3765-3781, doi:10.5194/angeo-26-3765-2008, 2008.

Kuo, F. S., Lue, H. Y., Fern, C. L., Röttger, J., Fukao, S., and Yamamoto, M.: Statistical characteristics of AGW wave packet propagation in the lower atmosphere observed by the MU radar, Ann. Geophys., 27, 3737-3753, doi:10.5194/angeo-27-37372009, 2009.

Kuo, F. S., Lue, H. Y., Fukao, S., and Nakamura, T.: Studies of gravity wave propagation in the mesosphere observed by MU radar, in preparation, 2012.

Nakamura, T., Tsuda, T., Yamamoto, M., Fukao, S., and Kato, S.: Characteristics of gravity waves in the mesosphere observed with the middle and upper atmosphere radar, 2, Propagation direction, J. Geophys. Res., 98, 8911-8923, 1993.

Sato, K. and Yamada, M.: Vertical structure of atmospheric gravity waves revealed by the wavelet analysis, J. Geophys. Res., 99, 20623-20631, 1994.

Serafimovich, A., Hoffmann, P., Peters, D., and Lehmann, V.: Investigation of inertia-gravity waves in the upper troposphere/lower stratosphere over Northern Germany observed with collocated VHF/UHF radars, Atmos. Chem. Phys., 5, 295-310, doi:10.5194/acp-5-295-2005, 2005. 
Tsuda, T., Kato, S., Yokoi, T., Inoue, T., Yamamoto, M., VanZandt, T. E., Fukao, S., and Sato, T.: Gravity waves in the mesosphere observed with the middle and upper atmosphere radar, Radio Sci., 26, 1005-1018, 1990.
Vincent, R. A. and Fritts, D. C.: A climatology of gravity wave motions in the mesopause region at Adelaide, Australia, J. Atmos. Sci., 44, 748-760, 1987. 